\title{
A Study on Design, Development, Implementation, and Evaluation of Severe Drug-Drug Interaction Alert System in ICU
}

mehrdad Karajizadeh

Shiraz University of Medical Sciences

Farid Zand

Shiraz University of Medical Sciences

Roxana Sharifian ( $\square$ sharifianr@sums.ac.ir)

Shiraz University of Medical Sciences

Afsaneh Vazin

Shiraz University of Medical Sciences

Najmeh Bayati

Shiraz University of Medical Sciences

\section{Research Article}

Keywords: Drug-Drug Interaction, Clinical Decision Support System, Knowledge Base, Medication Alerting system, Intensive Care Units

Posted Date: September 29th, 2021

DOl: https://doi.org/10.21203/rs.3.rs-913472/v1

License: (c) (1) This work is licensed under a Creative Commons Attribution 4.0 International License. Read Full License 


\section{Abstract \\ Background and objective:}

The overridden rate of Drug-Drug Interaction Alerts (DDIAs) in the Intensive Care Unit (ICU) is very high. Therefore, this study aimed to design, develop, implement, and evaluate a severe Drug-Drug Alert System (DDIAS) in ICU and measure the override rate of DDIAs.

\section{Methods}

This is a cross-sectional study for the design, development, implementation, and evaluation of severe DDIAs into a Computerized Provider Order Entry(CPOE) system in the ICUs of Nemazee general teaching hospitals in 2021. The patients exposed to the volume of DDIAs, acceptance and overridden of DDIAs, and usability of DDIAS have been collected.

\section{Results}

The knowledge base of DDIAS contains 9,809 severe DDIs. A total of 2672 medications were prescribed in the population study. The volume and acceptance rate for severe DDIAs were 81 and $97.5 \%$, respectively. However, the override rate was $2.5 \%$. The mean System Usability Scale (SUS) score of the DDIAS was 75.

\section{Conclusion}

This study demonstrated that the implementation of high-risk DDIAs at point of prescribing in ICU improved adherence to alerts. In addition, the usability of DDIAS was reasonable. Further studies are need to investigate the establishment of severe DDIAS and measure the physician's response to DDIAS on a larger scale.

\section{1- Introduction}

Drug-drug interactions (DDIs) occur when two or more drugs react with each other, which may cause to experience an unexpected side effect (fda.gov). DDIs are the most common cause of medical errors worldwide (1). The prevalence of DDIs in hospitalized patients ranges from 4.0 to $9.0 \%$ (2). The rate of DDIs in ICU setting are higher than in the general ward and outpatients(3). ICU patients are more prone to effects of DDIs due to polypharmacy and kidney and liver dysfunctions (4). Moreover, insufficient knowledge and mismanagement about DDIs among healthcare staff lead to elevated morbidity and mortality rates following adverse therapy and elevated time and costs in health systems (5-7). 
Computerized provider order entry (CPOE) is a promising technology that allows physicians to enter orders into a computer instead of handwriting them(8). CPOE can enhance completeness, legibility, and accessibility of medical prescription, however it cannot prevent DDIs without the clinical decision support system (CDSS) (9). The CDSS integrated into the CPOE system can assist the physicians in detecting DDIs at the point of prescribing (10). The physician attention is imperative to decrease and/or to inhibit DDI rates; however, DDIs rates are high. Likewise, in the ICU, approximately $90 \%$ of Drug-Drug Interaction Alerts (DDIA)s are neglected (11-13). On the hand, clinically irrelevant alerts are one of the main reasons of override DDIAs $(14,15)$. Also, patients can tolerate the adverse effects, thus physicians may decide to override alerts of DDIs (14). A previous study indicated that prescribers overrode a large proportion of highest-severity DDIAs in ICU $(15,16)$.

CDSS within CPOE assist the physician to prevent common medication errors in the health centers (17). Despite intensive effort to improve drug-drug interaction alert systems (DDIAS)s, override rate of this systems remain high (18). DDIAs increase the workload of healthcare staff. Nevertheless, previous studies showed several strategies to reduce irrelevant DDIAs, including applying the pharmacist recommendations to review DDIs, considering context factors of diagnosis, patient age and laboratory value $(19,20)$. DDIA systems reached high priority for implementation in ICU settings in Iran based on a previous Karajizadeh and et al.'s study (21). Here, the purpose of this study was to design, develop, implement, and evaluate severe DDIAs in the ICU. The results of our study will hopefully help to increase the consideration of DDIAs in the ICU setting, which may lead to diminishing side effects of overridden alerts.

\section{2- Material And Methods \\ 2 - 1 Study design}

This is a cross-sectional study and All methods was reported according to the Strengthening the Reporting of Observational Studies in Epidemiology (STROBE) Statement guideline.

\section{2-2 setting and participants, data collection}

The data was collected and assembled in ICUs of Nemazee general teaching hospital in southern Iran in 2021. All of the ICU systems managed by the same organization manners and rules. Electronic medical record (EMR) with CPOE has been used in the ICU of Nemazee general teaching hospital since 2015, without incorporation of DDIAs (22).

Inclusion criteria included patients over 18 years of age, hospitalization in the ICU, with high risk (risk rating X) DDIs(23). However, intermediate DDls risk (risk rating D, C, B, A)(23) was excluded.

\section{2-3 DDIA System}

\section{2-3-1 Knowledge base of severe DDIA system in ICU setting}


The research team extracted the severe DDIs with a risk rating of $X$ (avoid combination) from the Lexiinteract(24), including drug-class interaction and DDIs. Lexi-interact program have appropriate sensitivity and specificity for detect DDIs in ICU(25). The medications of the study were coded according to national drug coding system.

\section{2-3-2 Design and development of DDIA system}

The unified modeling language (UML) is a term in software engineering that is applied to provide a standard way to envision the design of a system(26). Here, common UML diagrams, including use case, activity, and sequence diagrams, were provided to visualize a DDIAS. A visual paradigm community edition software was used to visualize UML diagrams. Node.js(27) and MongoDB(28) were used to develop DDIAS.

\section{2-3-2-1 Use case diagram of DDIAS}

UML analyzes the use case diagram depictions to show interaction with a DDIA system (Fig. 1). Also, use case diagram shows various tasks which different users should consider them (Fig. 1).

\section{2-3-1-2 Activity diagram of severe DDIAS in ICU setting}

UML activity diagrams show workflows activities, stepwise actions of the DDIAS, and step-wise of DDIA and clinician response to this alerts (Fig. 2).

\section{2-3-1-3 Sequence diagram of DDIAS in ICU setting}

Also, UML sequence diagram shows, EMR, CPOE system, medication order entry system, message exchange sequences between health care professionals, and DDI detection engine arranged in time sequence (Fig. 3).

\section{2-3-1-4 Designing user interface of Severe DDIAS in ICU setting}

Our research team including, clinical pharmacists and health information management, concluded that user interface content should report a risk rating scale (severe DDI), recommendations for DDI management, and drug name interactions. Figure 4 shows a user interface of the DDIAS.

\section{2-3-3 Testing and Implementation DDIA system in ICU setting}

ICU physicians' representative and CDS committee in the hospital assessed and tested the DDIA system. All major bugs or modification in DDIA content have been fixed after testing the system. After approval of DDIA system by the CDS committee, all end-users (physician) have been trained to use the DDIA in a meeting and one by one. Finally, the DDIA system has been installed in ICUs of Nemazee hospitals. The unexpected bugs in the DDIA system were resolved every day.

\section{2-3-4 Evaluation of DDIA system performance in ICU setting}


The total number of fired severe DDIAs and physician's response to it has been recruited. The rate of acceptance and overriding of serious DDIs were calculated based on the following formula(29).

Acceptance Rate of DDI alerts $=\frac{A+B}{C} * 100$

Overriden rateof DDI alerts $=\frac{\mathrm{D}}{\mathrm{C}} * 100$
A: Count of clinician response to severe DDIAs (order current drug and discontinue existing order)
B: Count of clinician response to severe DDIAs (cancel the current order and keep existing order)
C: Total number of alerts serious DDIAs Fired
D: Count of clinician response to severe DDIAs (order current drug and keep existing order)

\section{2-3-5 Measurement of usability level of the DDIAS}

The system usability scale (SUS) questionnaire(30) was used to measure the usability of a severe DDIAS. The SUS questionnaire consists of 10 questions with five Likert scale options, ranging from strongly agree to disagree strongly. The SUS score was calculated based on Brooke et al. study(30). The SUS questionnaire is accessible in a supplementary file in Appendix 1.

\section{2-4 Statistical analysis}

The sample size method was convenient. All medication prescribed in ICUs was reviewed for three months after implementation DDIAS. Continuous variables are summarized as means and standard deviations, and categorical variables are presented with numbers and ratios. All statistical analysis was performed via SPSS version 24 .

\section{3- Result}

\section{3 - 1 Descriptive finding}

In this study the patients exposed to serious DDIA were $57 \pm 18$ years old. Forty-four percent of patients who were subject to DDIAs were male. The patients' characteristics are presented in Table 1. 
Table 1

Patient Characteristics who exposed severe drug-drug interaction alerts

\begin{tabular}{|c|c|c|}
\hline \multicolumn{2}{|c|}{ Variable } & Value \\
\hline \multicolumn{2}{|c|}{ Age $($ mean $\pm S D)$} & $56.96 \pm 18.40$ \\
\hline \multicolumn{2}{|c|}{ Height (mean \pm SD) } & $162.44 \pm 26.47$ \\
\hline \multicolumn{2}{|c|}{ Weight (mean \pm SD) } & $80 \pm 17.38$ \\
\hline \multirow[t]{2}{*}{ Sex } & Male (Frequency: Percent) & $11(44 \%)$ \\
\hline & Female (Frequency: Percent) & $15(56 \%)$ \\
\hline \multicolumn{2}{|c|}{ The mortality rate in ICU (Frequency: Percent) } & $6(24 \%)$ \\
\hline \multicolumn{2}{|c|}{ Length of stay in ICU (mean \pm SD) } & $19.64 \pm 17.41$ \\
\hline
\end{tabular}

\section{3 - 2 Knowledge Base Of Ddia System}

The knowledge base of DDIA system consists 9809 cases of DDI documented in the Lexi interact database (with risk rating, interaction effect, and patient management recommendation).

\section{3-3 Evaluation Performance Of Ddia System}

A total of 2,672 medications were ordered over the study period (three months after intervention DDIAS). Eighty-one severe DDIAs were recorded in ICU over the study period and the triggered unique DDIAs were 25. The most common triggering DDIAs were Potassium Chloride with Haloperidol, Potassium Chloride with Atropine, and Potassium Chloride with Ipratropium, respectively (Fig. 5).

The rate of acceptance for DDIASs was $97.5 \%$, while the override rate was $2.5 \%$. The metrics for DDIAs are shown in Table 2.

Table 2

drug-drug interaction alert system metrics

\begin{tabular}{|ll|}
\hline Metrics & Value \\
\hline Total medication order (frequency) & 2672 \\
\hline The volume of Severe DDIAs* (Frequency: Percent) & $81(3 \%)$ \\
\hline Acceptance rate of Severe DDIAs* (Frequency: Percent) & $79(97.5 \%)$ \\
\hline Overridden rate of Severe DDIAs* (Frequency: Percent) & $2(2.5 \%)$ \\
\hline * Drug-Drug Interaction alerts(DDIAs) & \\
\hline
\end{tabular}




\section{3-4 Evaluation Usability Of Ddia System}

Table 3 shows the results of the mean user responses regarding the usability of the context-aware DDIAs. Most of the users were eager to use DDIASs at point of prescribing (mean: 4.33; SD: 0.516). The mean SUS score of DDIAS was 75. The mean value of the usability statements is presented in Table 3.

Table 3

Evaluation usability of drug-drug interaction alert system

\begin{tabular}{|lcc|}
\hline Question Item & Mean & SD** \\
\hline I think I want to use the DDIAS* frequently. & 4.33 & 0.516 \\
\hline I found the DDIAS* unnecessarily complex. & 2.67 & 1.211 \\
\hline I thought the DDIAS* was easy to use. & 4.17 & 0.408 \\
\hline $\begin{array}{l}\text { I think that I would need the support of a technical person to be able to use the } \\
\text { DDIAS*. }\end{array}$ & 1.67 & 0.516 \\
\hline I found the various functions in the DDIAS* were well integrated. & 3.83 & 0.40 \\
\hline I thought there was too much inconsistency in the DDIAS*. & 2.17 & 0.983 \\
\hline I would imagine that most people would learn to use a DDIAS* very quickly. & 3.67 & 1.21 \\
\hline I found the DDIAS* cumbersome to use. & 2.17 & 0.98 \\
\hline I felt confident using the DDIAS*. & 4.17 & 0.40 \\
\hline I needed to learn many things before I could get going with the DDIAS*. & 2.00 & 0.89 \\
\hline SUS** Score & 75 & 12.04 \\
\hline * Drug-Drug Interaction Alert System(DDIAS) & & \\
** System Usability Scale (SUS) & & \\
*** Standard Deviation(SD) & & \\
\hline
\end{tabular}

\section{4- Discussion}

DDIs can cause adverse drug event in ICU population. DDIA system have indicated their potential to decrease DDIs. However, override rate of DDIA in ICU setting is high(12). Therefore, the primary purpose of this study was to design, development and evaluation of serious DDIASs in the ICU. To our knowledge, this is the first study to develop and implement a DDIAS in the CPOE in the ICU of an Iranian hospital. DDIAS interface contents included how the physician reacts to alerts that recommend ordering or canceling current medication against keeping or discontinuing existing order, or overriding drug alerts (order current drug and keep existing order). This study demonstrated that the acceptance rate of triggered DDIAS rates is high and the usability level of the DDIASs is in reasonable level. 
In this study, knowledge-based included severe DDIs for ICU setting. In contrast, some studies showed that knowledge-based of routine DDIASs contains any potential DDI without intensity leveling (32). Our results are consistent with Paterno et al. study (33), which showed that classification of DDIs by severity increases compliance rate (33). Also, Knight et al. indicated that removing moderate severity DDIAs decreases the override rate, albeit they remained high (34). There are several potential reasons for the low rate of overridden DDIAs in our study; Firstly, triggered DDIAs at the exact point and time of prescribing of the order by the physician in CPOE system. Secondly, actionable recommendations when the physician encounters the serious DDIAs. Third, an ICU administration and team interested in using health information technology in point of care (35). Fourth, the already working structured CPOE system that has been implemented since 2015 in the ICU of Nemazee general teaching hospital. Therefore, health care providers are familiar with $\mathrm{CPOE}$, and all medication orders were entered electronically by the physicians. Fifth, in this study we used Node.js and MongoDB for implement DDIAS that improved the speed of DDIA system. Sixth, grading DDIAs may improve the acceptance of this system (36). Seventh, in this system development process, we considered five right (the right information, to the right person, in the right CDS intervention format, through the right channel, at the right time in workflow) of CDSS (37). Eighth, our system was its integration into a homegrown CPOE and its especial design for the ICU setting. In contrast, some studies indicated that overridden rate of DDIAs remains high (range from $40.1 \%$ to 96.2 $\%)(12,14-16,38-43)$. However, most physicians actions over the overridden recommendations were appropriate (39). Even though a small fraction of serious DDIAs were overridden in this study, some overriding of DDIAs are acceptable in the ICU because patients are closely observed $(13,44)$. The significance of these findings is that the serious DDIAs and actionable recommendations contribute to reduce the overridden rate of DDIA in the ICU $(14,45)$. The clinicians should not follow DDIAs recommendations without considering the total risk-benefit balance for a DDIs. Here, severe DDIAs have been stored in an electronic database. However, without this system, DDIs collection data could be incomplete. Therefore, a DDIA system is imperative for decreasing drug error events. The results of this study could be considered as a solution to reduce the rate of overriding of DDIAs.

The usability of the DDIASs was at a reasonable level. Therefore, users like to use safe and usable warning systems in the field of DDIs. The results of our study are in line with Kavumas et al. (46), which showed the high usability levels of EMR systems implemented in sub-Saharan Africa. On the contrary, some reports showed lower usability rates of the $\operatorname{EMR}(47,48)$. An important implication of these findings is that end-user's participation in designing the DDIASs interface may play an essential role in improving the usability of this system. Also, in this system, consistency medication terminology, visual cues for severity rate of DDIs, and minimal text was used that may impact on improving on usability of DDIAS(49). Nevertheless, the usability of DDIASs is not excellent. Clinical information systems(CIS) have some unintended consequences, including workload, ignoring the role of the clinical pharmacists, alter in the authority structure, and overdependence on the technology $(50,51)$.

The most important limitation of this study was the sample size. This system brings up a novel approach to DDI that could not be evaluated by our team in a large population as there is no mandating rule for use of DDIAs in the health systems in Iran. In this study, adverse drug events due to override-triggered DDIs 
were not investigated because of the constraining time and source of the project. We also must consider that our study could have been affected by location bias and suffered from a lack of randomization design.

\section{5- Conclusions}

This study completed the design, development, and implementation of severe DDIASs in the ICU. Our systems alerts lead to decreased overridden rates of DDIAs in ICU. The implementation of the DDIAS in other educational hospitals at the national level seems to be feasible. The following steps of our research are to investigate the establishment of severe DDIAS and measure the physician's response to DDIAS on a larger scale.

\section{Abbreviations}

CDSS: Clinical Decision Support System, DDI: Drug-Drug Interaction, DDIA: Drug-Drug interaction alert. DDIAS: Drug-Drug interaction alert system. EMR: Electronic Medical Records, CPOE: Computerized Provider Order Entry, EHR: Electronic Health Records, ADE: Adverse Drug Events, UML: Unified Modeling Language, SUS: System Usability Scale, ICU: Intensive Care Unit, DDI: Drug- Drug Interaction, UCD: User Centered Design.

\section{Declarations}

\section{Ethics approval}

The study was approved by the local Institutional Review Board (IRB); ethics committee of Shiraz University of Medical Science (Approval ID: IR.SUMS.REC.1398.1046).

\section{Confidentiality}

According to the SUMS agreement, anonymity of patients was maintained by de-identification of data. Only researchers or authorized personnel can access the data. To comply with SUMS and Nemazee hospital data security regulations, only research team can access patients and providers' information. SUMS and Nemazee Hospital security department approved the investigation protocol. Only trained and authorized users had access to DDIA system.

\section{Informed consent}

Not applicable.

\section{Acknowledgments}

This article was extracted from a doctoral thesis (research ID: 97-01-07-19299) supported by the Research Vice-Chancellor of Shiraz University of Medical Sciences. The authors are thankful for Research 
Vice-Chancellor of Shiraz University of Medical Sciences for financially supporting this project.

\section{Competing Interests}

The author declares that there is no potential conflict of interest with respect to the research, authorship or publication of this article.

\section{Author's Contribution}

MK: conceptual and design of study, data acquisition, data analysis, drafting and revision of paper. FZ: contributed to conceiving and design of the study, interpretation of data, commented on drafts, and made significant revisions to the paper. AV: contributed to the design of the study, interpretation of data, commented on the draft, and made significant revisions to the paper. RSH: contributed to the design of the study, statistical analysis, commented on drafts, and made significant revisions to the paper. NB: contributed to the design and implementation system, interpretation of data, commented on drafts, and made significant revisions to the paper.

\section{Funding}

This study was supported by the Shiraz University of Medical Science in the context of a dissertation project (Grant Number 97-01-07-19299).

\section{Data Availability Statement}

All relevant data is provided in the article, further required can be directed to the corresponding author (sharifianr@sums.ac.ir).

\section{References}

1. Karthikeyan, M., Balasubramanian, T., Khaleel, M., Sahl, M. \& Rashifa, P. A systematic review on medication errors. International Journal of Drug Development and Research, 7 (4), 9-11 (2015).

2. Gonzaga de Andrade Santos, T. N. et al. Prevalence of clinically manifested drug interactions in hospitalized patients: A systematic review and meta-analysis. PLoS One, 15 (7), e0235353-e (2020).

3. Cullen, D. J. et al. Preventable adverse drug events in hospitalized patients: a comparative study of intensive care and general care units. Crit Care Med, 25 (8), 1289-1297 (1997).

4. Papadopoulos, J. \& Smithburger, P. L. Common drug interactions leading to adverse drug events in the intensive care unit: management and pharmacokinetic considerations. Critical care medicine, 38, S126-S35 (2010).

5. Chen, Y-F. et al. Incidence and possible causes of prescribing potentially hazardous/contraindicated drug combinations in general practice. Drug Saf, 28 (1), 67-80 (2005).

6. Hines, L. E. \& Murphy, J. E. Potentially harmful drug-drug interactions in the elderly: a review. The American journal of geriatric pharmacotherapy, 9 (6), 364-377 (2011). 
7. Magro, L., Moretti, U. \& Leone, R. Epidemiology and characteristics of adverse drug reactions caused by drug-drug interactions. Expert opinion on drug safety, 11 (1), 83-94 (2012).

8. Ozdas, A. \& Miller, R. Care provider order entry (CPOE): a perspective on factors leading to success or to failure. Yearbook of medical informatics, 16 (01), 128-137 (2007).

9. Berner, E. S. Clinical decision support systems: state of the art. AHRQ publication, 90069, 1-26 (2009).

10. Helmons, P. J., Suijkerbuijk, B. O., Nannan Panday, P. V. \& Kosterink, J. G. Drug-drug interaction checking assisted by clinical decision support: a return on investment analysis. Journal of the American Medical Informatics Association, 22 (4), 764-772 (2015).

11. Wong, A. et al. Prospective evaluation of medication-related clinical decision support over-rides in the intensive care unit. BMJ quality \& safety, 27 (9), 718-724 (2018).

12. Wong, A. et al. Evaluation of medication-related clinical decision support alert overrides in the intensive care unit. Journal of critical care, 39, 156-161 (2017).

13. Nanji, K. C. et al. Medication-related clinical decision support alert overrides in inpatients. Journal of the American Medical Informatics Association, 25 (5), 476-481 (2017).

14. Wright, A. et al. Structured override reasons for drug-drug interaction alerts in electronic health records. Journal of the American Medical Informatics Association, 26 (10), 934-942 (2019).

15. Grizzle, A. J. et al. Reasons Provided by Prescribers When Overriding Drug-Drug Interaction Alerts.American Journal of Managed Care. 2007;13(10).

16. Edrees, H., Amato, M. G., Wong, A., Seger, D. L. \& Bates, D. W. High-priority drug-drug interaction clinical decision support overrides in a newly implemented commercial computerized provider orderentry system: Override appropriateness and adverse drug events. Journal of the American Medical Informatics Association: JAMIA, 27 (6), 893-900 (2020).

17. Nabovati, E. et al. Information technology-based interventions to improve drug-drug interaction outcomes: a systematic review on features and effects. Journal of medical systems, 41 (1), 1-17 (2017).

18. Bryant, A., Fletcher, G. \& Payne, T. Drug interaction alert override rates in the Meaningful Use era. Applied clinical informatics, 5 (03), 802-813 (2014).

19. Chou, E. et al. Designing and evaluating contextualized drug-drug interaction algorithms. JAMIA open, 4 (1), ooab023 (2021).

20. Pirnejad, H. et al. Preventing potential drug-drug interactions through alerting decision support systems: A clinical context based methodology. International journal of medical informatics, 127, 18-26 (2019).

21. Karajizadeh, M., Zand, F., Vazin, A., Nasiri, M. \& Sharifian, R. Identification and Prioritization of Clinical Decision Support Functionalities Built Within A Computerized Provider Order Entry System. Health Scope. 2021;10(1). 
22. Khammarnia, M., Sharifian, R., Zand, F., Keshtkaran, A. \& Barati, O. Designing computerized provider order entry software in Iran: The nurses' and physicians' viewpoints. CIN: Computers, Informatics, Nursing, 34 (9), 413-420 (2016).

23. Shafiekhani, M., Vazin, A., Karimi, S. \& Davarpanah, M. Evaluating drug interactions, adverse drug reactions, and level of adherence to highly active antiretroviral therapy regimen amongst HIV-positive patients who referred to an AIDS healthcare center in Fars, southern Iran: the first multifaceted study from Iran. HIV and AIDS Review, 16, 24-31 (2017).

24. Shojania, K. G. et al. The effects of on-screen, point of care computer reminders on processes and outcomes of care.The Cochrane database of systematic reviews. 2009(3):Cd001096.

25. Reis, A. M. M. \& Cassiani, S. H. D. B. Evaluation of three brands of drug interaction software for use in intensive care units. Pharmacy world \& science, 32 (6), 822-828 (2010).

26. Booch, G. The unified modeling language user guide (Pearson Education India, 2005).

27. Tilkov, S. \& Vinoski, S. Node. js: Using JavaScript to build high-performance network programs. IEEE Internet Computing, 14 (6), 80-83 (2010).

28. Chodorow, K. MongoDB: the definitive guide: powerful and scalable data storage (" O'Reilly Media, Inc.", 2013).

29. McGreevey, J. D. III, Mallozzi, C. P., Perkins, R. M., Shelov, E. \& Schreiber, R. Reducing alert burden in electronic health records: state of the art recommendations from four health systems. Applied clinical informatics, 11 (01), 001-012 (2020).

30. Brooke, J. SUS-A quick and dirty usability scale. Usability evaluation in industry, 189 (194), 4-7 (1996).

31. Khammarni, M. et al. Prescribing errors in two ICU wards in a large teaching hospital in Iran. The International journal of risk \& safety in medicine, 27 (4), 169-175 (2015).

32. Wright, A. et al. Reduced Effectiveness of Interruptive Drug-Drug Interaction Alerts after Conversion to a Commercial Electronic Health Record. J Gen Intern Med, 33 (11), 1868-1876 (2018).

33. Paterno, M. D. et al. Tiering drug-drug interaction alerts by severity increases compliance rates. Journal of the American Medical Informatics Association: JAMIA, 16 (1), 40-46 (2009).

34. Knight, A. M. et al. The Effect of Eliminating Intermediate Severity Drug-Drug Interaction Alerts on Overall Medication Alert Burden and Acceptance Rate. Appl Clin Inform, 10 (5), 927-934 (2019).

35. Niknam, F., Khammarnia, M., Zand, F., Fallahnejad, E. \& Sharifian, R. Evaluating physicians and nurses' satisfaction with computerized physician order entry system in Iran. Journal of Health Management \& Informatics, 5 (2), 51-56 (2018).

36. Tilson, H. et al. Recommendations for selecting drug-drug interactions for clinical decision support. Am J Health Syst Pharm, 73 (8), 576-585 (2016).

37. Olakotan, O. O. \& Mohd Yusof, M. The appropriateness of clinical decision support systems alerts in supporting clinical workflows: A systematic review. Health Informatics Journal, 27 (2), 14604582211007536 (2021). 
38. Duke, J. D., Li, X. \& Dexter, P. Adherence to drug-drug interaction alerts in high-risk patients: a trial of context-enhanced alerting. J Am Med Inform Assoc, 20 (3), 494-498 (2013).

39. Poly, T. N., Islam, M. M., Yang, H. C. \& Li, Y. J. Appropriateness of Overridden Alerts in Computerized Physician Order Entry: Systematic Review. JMIR medical informatics, 8 (7), e15653 (2020).

40. Cho, I., Lee, Y., Lee, J. H. \& Bates, D. W. Wide variation and patterns of physicians' responses to drugdrug interaction alerts. International journal for quality in health care: journal of the International Society for Quality in Health Care, 31 (2), 89-95 (2019).

41. Humphrey, K. et al. An Investigation of Drug-Drug Interaction Alert Overrides at a Pediatric Hospital. Hospital pediatrics, 8 (5), 293-299 (2018).

42. Yeh, M. L., Chang, Y. J., Wang, P. Y., Li, Y. C. \& Hsu, C. Y. Physicians' responses to computerized drugdrug interaction alerts for outpatients. Computer methods and programs in biomedicine, 111 (1), 1725 (2013).

43. Mille, F. et al. Analysis of overridden alerts in a drug-drug interaction detection system. International journal for quality in health care: journal of the International Society for Quality in Health Care, 20 (6), 400-405 (2008).

44. Bakker, T. et al. The effect of ICU-tailored drug-drug interaction alerts on medication prescribing and monitoring: protocol for a cluster randomized stepped-wedge trial. BMC Medical Informatics and Decision Making, 19 (1), 159 (2019).

45. Russ, A. L., Zillich, A. J., McManus, M. S., Doebbeling, B. N. \& Saleem, J. J. Prescribers' interactions with medication alerts at the point of prescribing: A multi-method, in situ investigation of the human-computer interaction. International Journal of Medical Informatics, 81 (4), 232-243 (2012).

46. Kavuma, M. The Usability of Electronic Medical Record Systems Implemented in Sub-Saharan Africa: A Literature Review of the Evidence. JMIR Hum Factors, 6 (1), e9317-e (2019).

47. Bloom, B. M., Pott, J., Thomas, S., Gaunt, D. R. \& Hughes, T. C. Usability of electronic health record systems in UK EDs. Emergency medicine journal: EMJ, 38 (6), 410-415 (2021).

48. Melnick, E. R. et al. The Association Between Perceived Electronic Health Record Usability and Professional Burnout Among US Physicians. Mayo Clinic proceedings. 2020;95(3):476 - 87.

49. Payne, T. H. et al. Recommendations to improve the usability of drug-drug interaction clinical decision support alerts. Journal of the American Medical Informatics Association: JAMIA, 22 (6), 1243-1250 (2015).

50. Campbell, E. M., Sittig, D. F., Ash, J. S., Guappone, K. P. \& Dykstra, R. H. Types of unintended consequences related to computerized provider order entry. Journal of the American Medical Informatics Association: JAMIA, 13 (5), 547-556 (2006).

51. Ash, J. S., Sittig, D. F., Campbell, E. M., Guappone, K. P. \& Dykstra, R. H. Some unintended consequences of clinical decision support systems. AMIA Annual Symposium proceedings AMIA Symposium. 2007;2007:26-30.

\section{Figures}



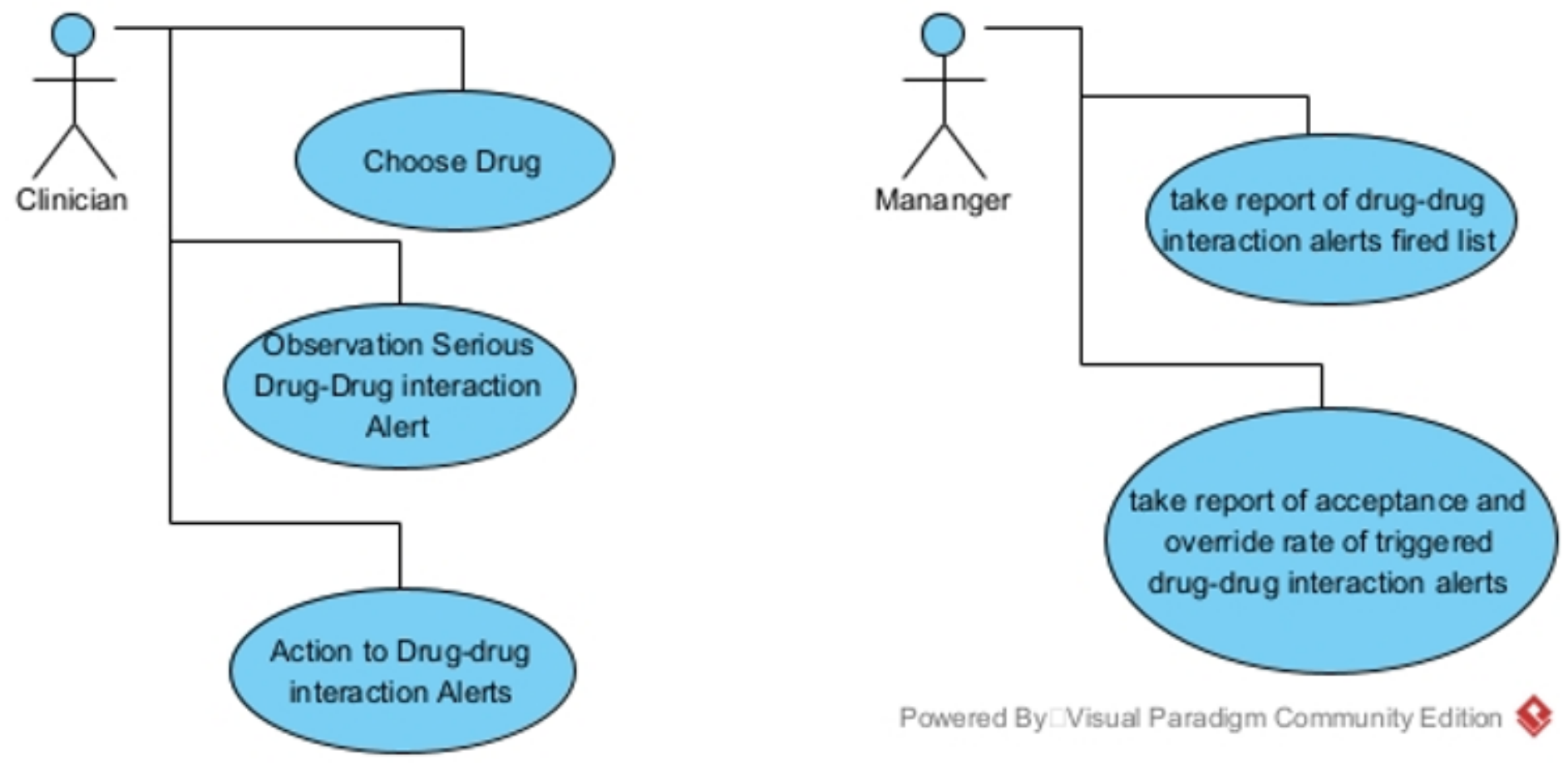

Figure 1

Use Case Diagram of Drug-Drug Interaction Alerts System 


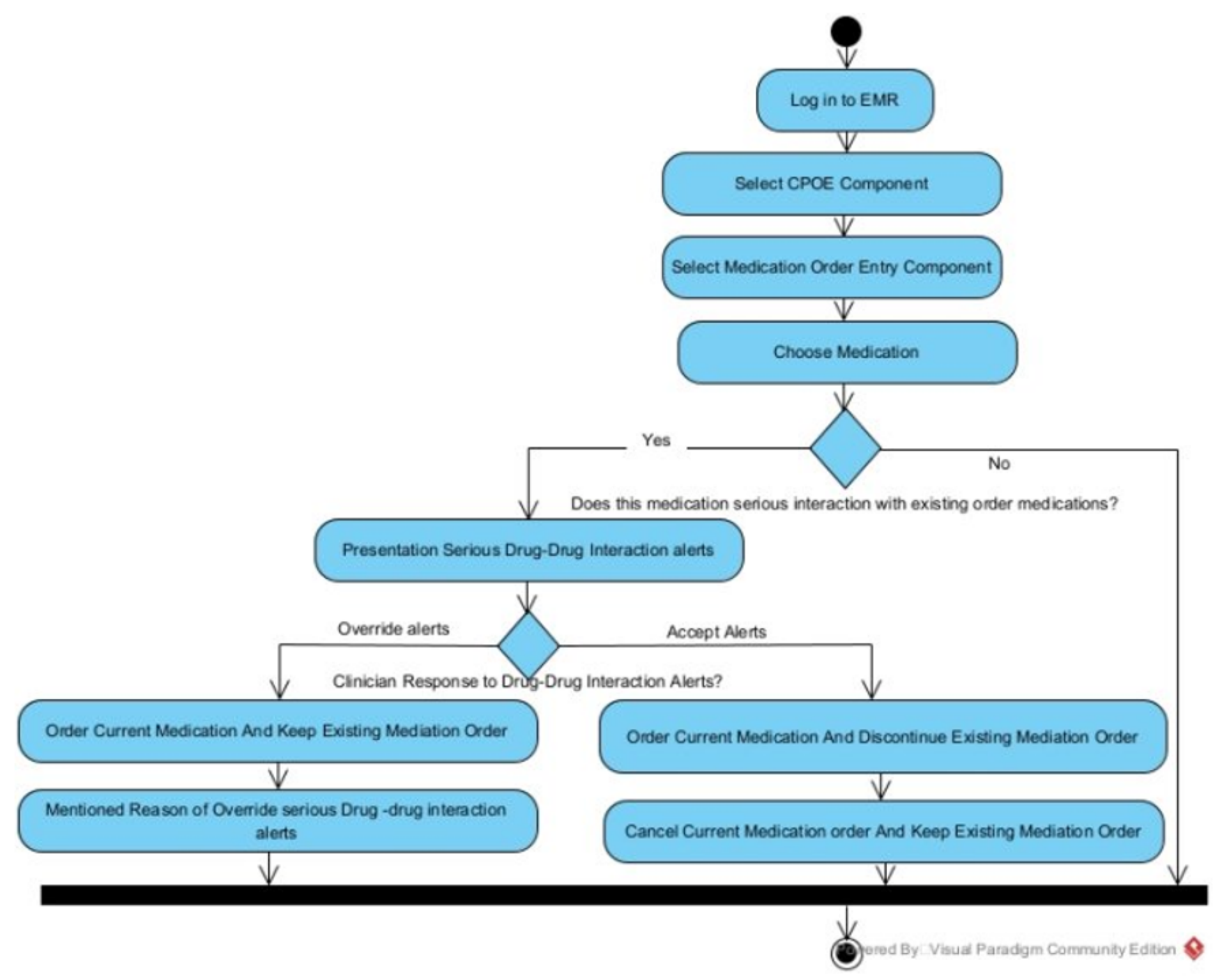

\section{Figure 2}

Activity Diagram of Serious Drug-Drug Interaction Alerts System 


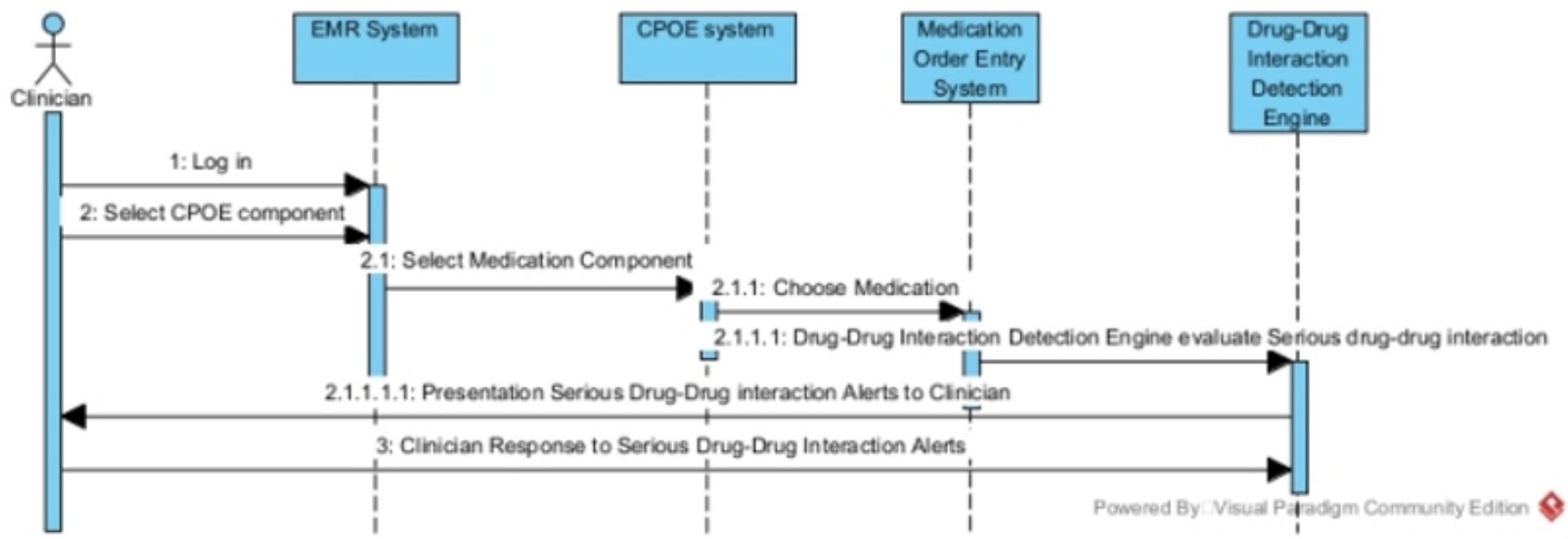

\section{Figure 3}

Sequence Diagram of Drug-Drug Interaction Alerts System

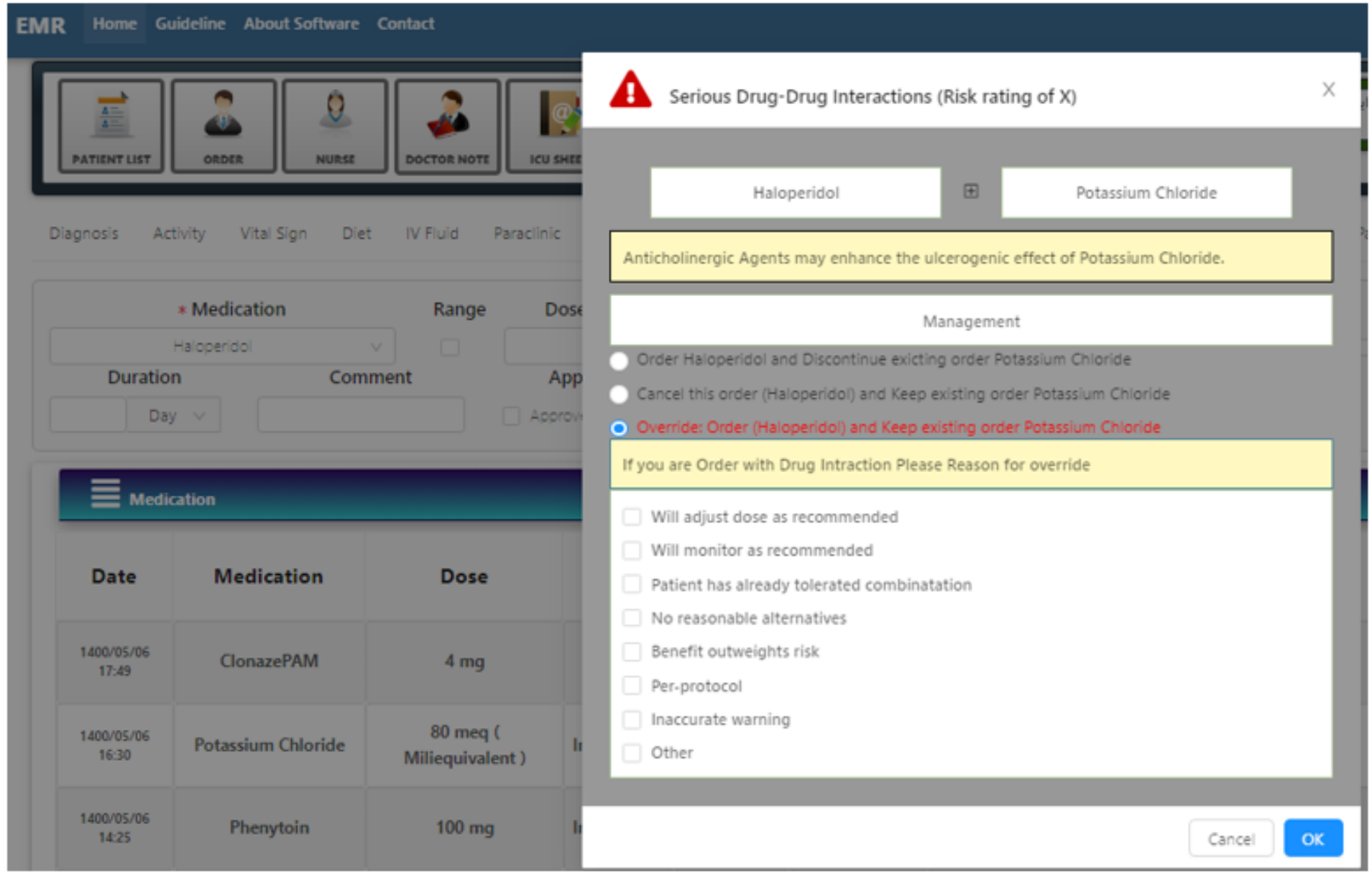

Figure 4

User interface of serious drug-drug alert system in ICU 


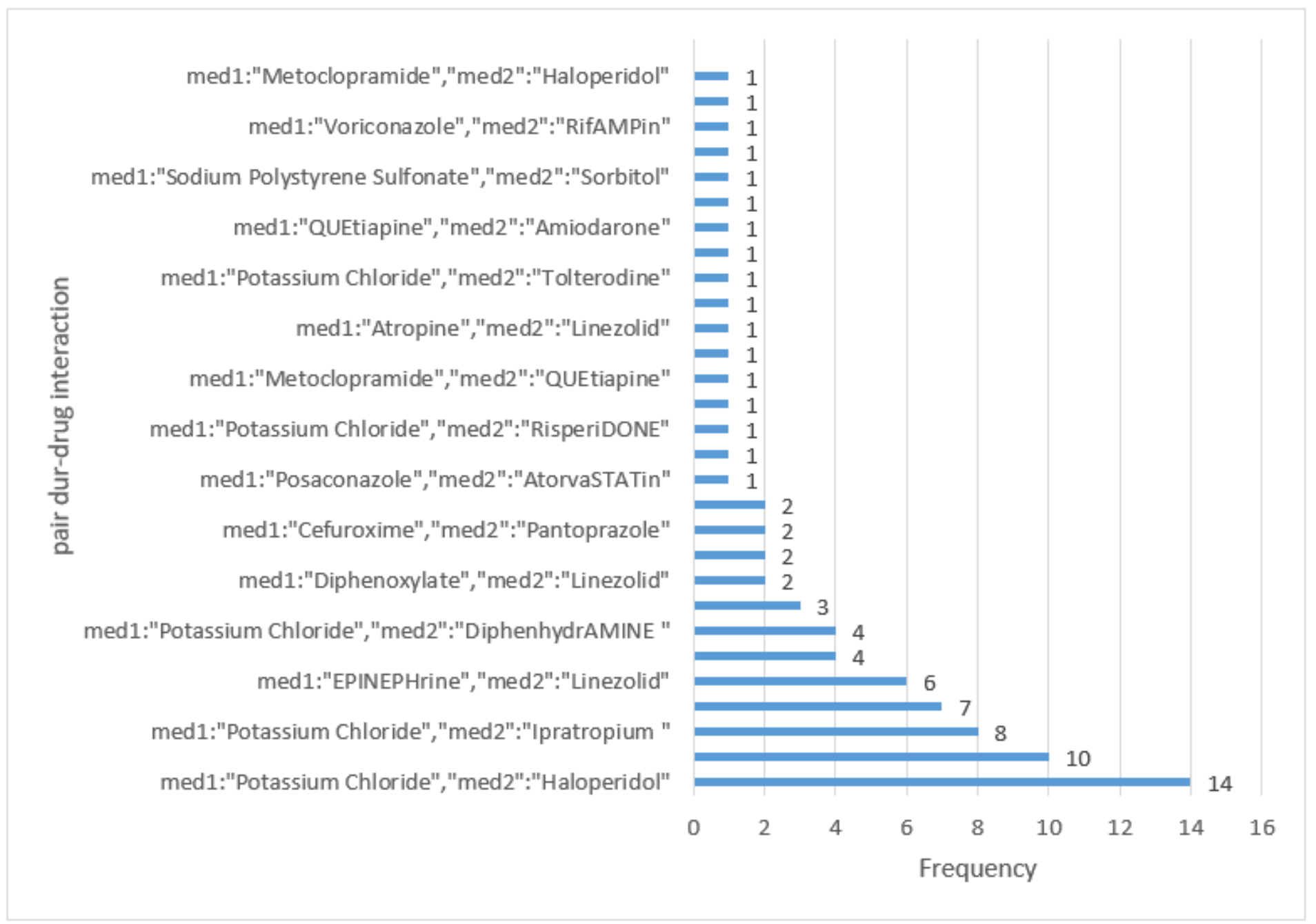

\section{Figure 5}

Triggered serious drug-drug interaction alerts in ICU

\section{Supplementary Files}

This is a list of supplementary files associated with this preprint. Click to download.

- Supplementary.docx 